ESAIM: PROCEEDINGS, March 2012, Vol. 35, p. 167-173

Fédération Denis Poisson (Orléans-Tours) et E. Trélat (UPMC), Editors

\title{
TRANSITION DE DÉPIÉGEAGE ÉLASTIQUE DE VORTEX SUPRACONDUCTEURS *
}

\author{
Enrick Olive ${ }^{1}$, Nicolas Di Scala ${ }^{1}$, Yves Lansac ${ }^{1}$, Yaouen Fily ${ }^{2}$ et Jean-Claude \\ SORET $^{1}$
}

\begin{abstract}
Résumé. Nous présentons des résultats de simulations numériques à deux dimensions sur des réseaux de vortex dans les supraconducteurs que l'on met en mouvement dans un potentiel aléatoire. On étudie la dynamique des vortex au seuil de dépiégeage $F_{c}$ dans le cas d'un faible désordre à température nulle. Les régimes élastiques au seuil de dépiégeage sont analysés dans le cadre des transitions de phase continues (transitions du second ordre). La réponse en vitesse $v$ à la force d'entraînement $F$ se comporte comme $v \sim\left(F-F_{c}\right)^{\beta}$ au voisinage immédiat du seuil de dépiégeage. Dans la région critique obtenue pour différentes grandes tailles du système simulé, nous mesurons l'exposant critique $\beta=0.27 \pm 0.04$.
\end{abstract}

\begin{abstract}
We present 2D numerical simulation results of superconductor vortex lattices driven over a random disorder. The vortex dynamics at the depinning threshold $F_{c}$ is studied at zero temperature in the case of weak disorder. The dynamics is elastic and the depinning transition is analysed in the framework of a second order phase transition where the velocity response $v$ to the driving force $F$ behaves like $v \sim\left(F-F_{c}\right)^{\beta}$. The analysis of the critical region of several large lattice sizes leads to the result that $\beta=0.27 \pm 0.04$.
\end{abstract}

\section{INTRODUCTION}

On s'intéresse à une large classe de systèmes qui présentent les caractéristiques communes suivantes : l'existence d'un état d'équilibre stable ordonné en l'absence de désordre, l'existence d'un domaine élastique (force de rappel proportionnelle aux déplacements par rapport à l'équilibre) pour de faibles déplacements, et l'irrégularité du milieu ambiant caractérisée par l'existence d'un potentiel désordonné gelé, c'est-à-dire figé aux échelles de temps caractéristiques du système. Il se joue donc une compétition entre l'élasticité de la structure qui tend à imposer un ordre parfait et le potentiel aléatoire qui tend à piéger et donc à déformer à plus ou moins grande échelle cet ordre parfait. Les systèmes considérés sont soit des structures périodiques qui peuvent être les réseaux de vortex dans les supraconducteurs, les ondes de densité de charge dans les métaux, le cristal de Wigner, ou les collö̈des, soit des variétés (appelées aussi interfaces) qui peuvent être les parois de domaine magnétique, la propagation de fracture dans les matériaux ou la dynamique d'une ligne de contact. Nous nous concentrons ici sur les structures périodiques (essentiellement les réseaux de vortex dans les supraconducteurs).

Selon l'intensité et/ou la concentration des pièges on distingue qualitativement le piégeage dit faible du piégeage dit fort. En piégeage faible l'élasticité domine le désordre et les déformations du système sont faibles par rapport

\footnotetext{
* Nous remercions vivement le Cluster CaSciModOT d'Orléans-Tours pour le temps de calcul dont nous avons pu disposer sur le Centre de Calcul Scientifique de la Région Centre - France

${ }^{1}$ LEMA, UMR 6157, Univ. F. Rabelais-CNRS-CEA, Parc de Grandmont, 37200 Tours, France; e-mail : olive@phys.univ-tours.fr

2 Department of Physics, Syracuse University, Syracuse, New York 13244, USA
}

(C) EDP Sciences, SMAI 2012 
à l'équilibre. Au contraire, on parle de piégeage fort si le désordre domine l'élasticité amenant la structure à se déformer de manière importante pour optimiser son énergie. Des déchirures dans la structure périodique peuvent alors se produire et l'approximation élastique de l'énergie du système n'est plus pertinente. Des modèles introduisant des déformations plastiques deviennent alors nécessaires. Pour illustrer ces deux régimes de piégeage nous présentons Fig.1 les configurations obtenues par simulation numérique à température nulle d'un réseau 2D de vortex. La triangulation de Delaunay laisse apparaître qu'en désordre faible (Fig. 1a) l'ordre topologique du

(a)

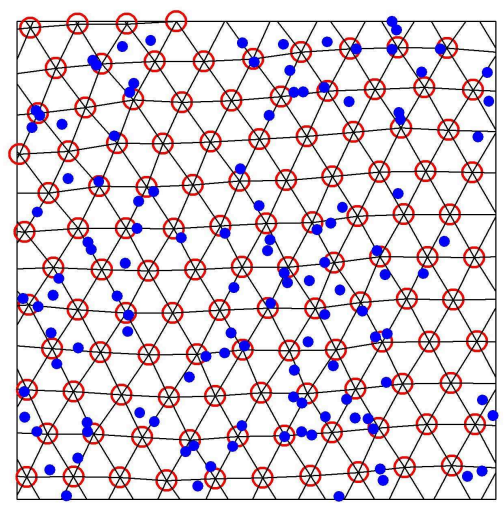

(b)

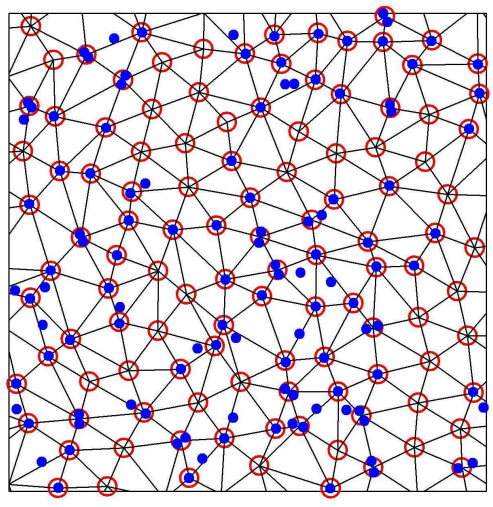

Figure 1. Configuration d'un réseau 2D de vortex : a) en présence d'un désordre faible ordre topologique conservé; b) en présence d'un désordre fort - ordre topologique détruit. Les cercles rouges représentent les vortex, les points bleus les pièges; la triangulation de Delaunay est représentée en noir pour aider à visualiser les défauts.

réseau est conservé tandis qu'il est détruit en piégeage fort (Fig. 1b).

Lorsque ces systèmes sont soumis à une force extérieure $F$, il existe une valeur critique $F_{c}$ séparant deux phases distinctes : une phase piégée (pour $F<F_{c}$ ) où la vitesse moyenne du système est nulle, et une phase en mouvement dite dépiégée (pour $F>F_{c}$ ) avec une vitesse moyenne finie. Pour les structures périodiques, deux types de dépiégeage génériques sont alors observés : le dépiégeage plastique lorsque le désordre domine l'élasticité (piégeage fort), caractérisé par un dépiégeage doux du système avec un écoulement plastique montrant notamment la coexistence de particules piégées et en mouvement, et le dépiégeage élastique lorsque l'élasticité domine le désordre (piégeage faible) qui est caractérisé par un dépiégeage brutal du système avec un écoulement élastique (en bloc) des particules. Fig. 2 illustre ces caractéristiques dynamiques. D’un point de vue théorique seul le dépiégeage de structures pour lesquelles le champ de déplacement est de dimension $N=1$ est correctement compris, et uniquement dans le cas élastique. En effet, dans le contexte des ondes de densité de charge le dépiégeage au seuil a été analysé [1] dans le cadre des phénomènes critiques à l'équilibre où la vitesse $v$ de la structure est le paramètre d'ordre et la force réduite $f=\left(F-F_{c}\right) / F_{c}$ est le paramètre de contrôle. Des travaux analytiques et numériques ultérieurs ont développé cette analogie pour définir des classes d'universalité et des exposants critiques (voir par exemple la Ref. [2] et les références qu'elle contient). Dans l'analogie avec une transition de phase continue (dite du second ordre) la vitesse de la structure élastique à température nulle s'annule à la force critique $F_{c}$ comme $v \sim f^{\beta}$ avec $\beta<1$. A l'heure actuelle, il n'existe pas de théorie satisfaisante pour $N=2$ (cas des réseaux de vortex et des colloïdes notamment) décrivant le dépiégeage élastique ou plastique. En particulier, la nature de la transition de dépiégeage plastique reste encore débattue : transition discontinue du premier ordre avec hystérésis, ou transition continue du second ordre avec lois d'échelle et exposants critiques. 
(a)
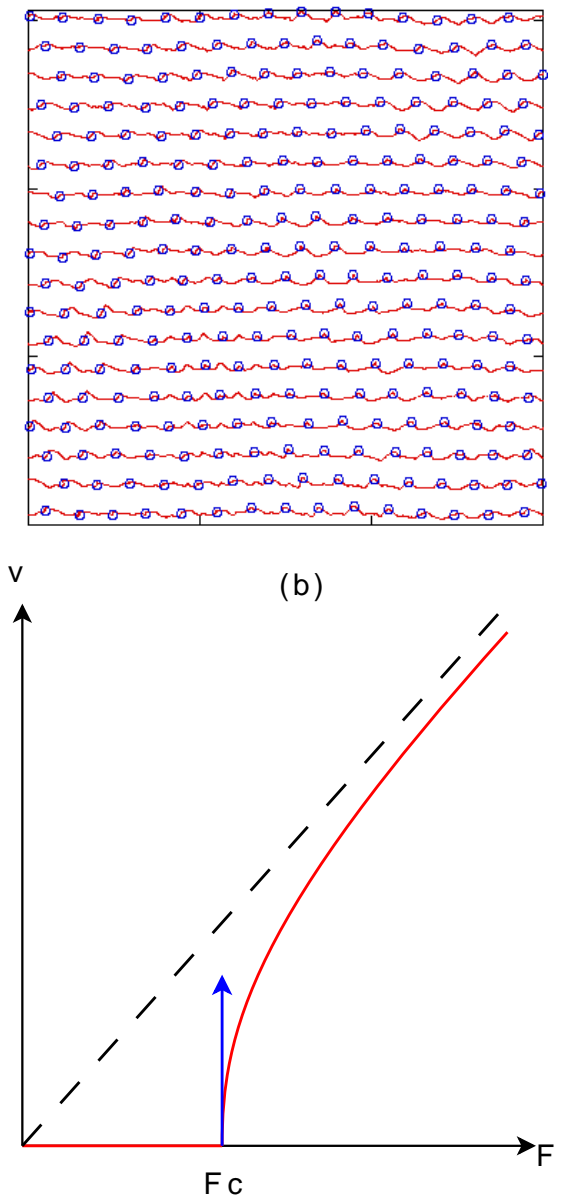

(c)
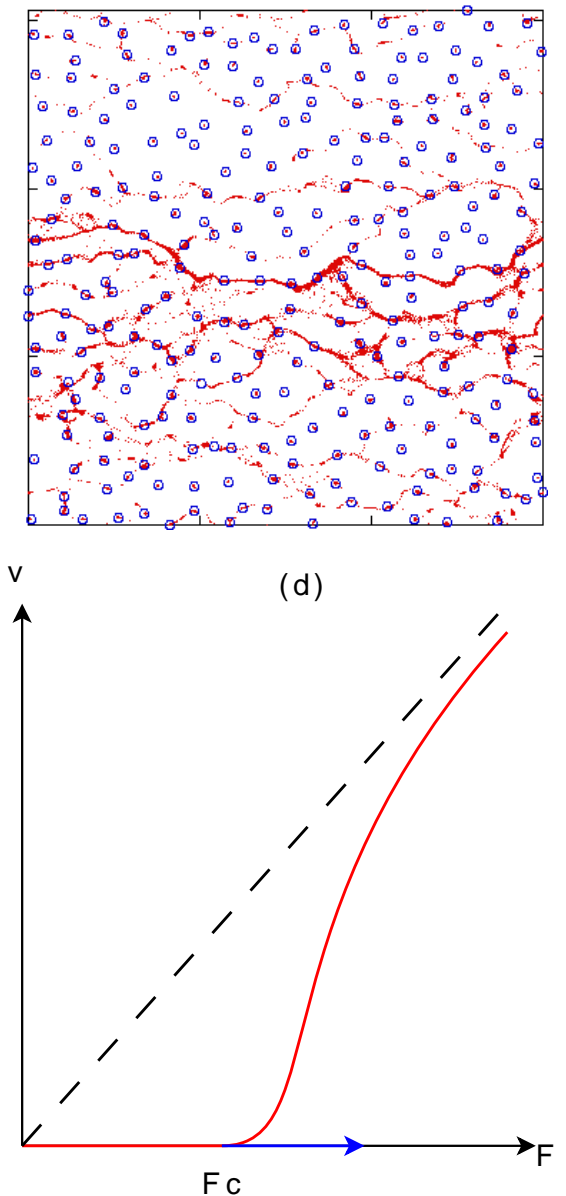

Figure 2. Dépiégeages élastique et plastique : trajectoires (en rouge) au seuil de dépiégeage et instantané des positions des vortex (cercles bleus) dans le cas élastique (a) et dans le cas plastique (c). Courbe vitesse-force : (b) dépiégeage brutal en $F_{c}$ dans le cas élastique; (d) dépiégeage doux en $F_{c}$ dans le cas plastique.

Concernant la transition de dépiégeage élastique, les simulations numériques dans un espace de dimension $d=2$ montrent des transitions du second ordre avec pour les interfaces $\beta \approx 1 / 3$ [3], tandis que pour les structures périodiques des exposants très variés ont été trouvés. $\beta \approx 2 / 3$ est mesuré pour les ondes de densité de charge [4], le cristal de Wigner [5] et pour les colloïdes [6], tandis que d'autres travaux ont trouvé $\beta \approx 0.5$ [7] et $\beta=0.92 \pm 0.01[8]$ pour des colloïdes, et $\beta=0.35[9]$ pour des systèmes de type stripes.

Dans ce papier nous abordons la complexité de ces systèmes à travers des simulations numériques à grande échelle de la dynamique à température nulle de réseaux 2D de vortex dans les supraconducteurs (donc $N=2$ et $d=2$ ) entraînés dans un potentiel désordonné. Nous étudions le dépiégeage élastique obtenu en désordre faible - pour les phases haute vitesse de vortex 3D en désordre faible voir Ref. [10], et pour les phases au seuil de dépiégeage plastique en désordre fort voir Refs. [11,12]. Plusieurs tailles de système ont été étudiées au voisinage du seuil de dépiégeage et les résultats montrent une transition de phase continue (second ordre). L'exposant 
critique $\beta$ de la vitesse est mesuré et est comparé aux valeurs trouvées dans des systèmes similaires.

\section{ModÈle NumÉRIQUE}

Nous étudions $N_{v}$ vortex dits d'Abrikosov dans les supraconducteurs de type II et interagissant avec $N_{p}$ pièges placés aléatoirement dans le plan $(x, y)$. Nous considérons la limite de London $\lambda_{L} \gg \xi$, où $\lambda_{L}$ est la profondeur de pénétration magnétique et $\xi$ la longueur de cohérence du supraconducteur, c'est-à-dire que nous traitons les vortex comme des particules ponctuelles. A température nulle nous intégrons les équations du second ordre de Newton pour chaque vortex $i$ à la position $\mathbf{r}_{i}$. La force totale sur chaque vortex inclut un terme de frottement visqueux $\eta \frac{d \mathbf{r}_{i}}{d t}$ où $\eta$ est le coefficient de viscosité, une force d'entraînement $\mathbf{F}^{L}=F \hat{\mathbf{x}}$ dans la direction $x$ (force dite de Lorentz due à un courant appliqué aux bornes du supraconducteur), ainsi que les forces conservatives issues des interactions vortex-vortex $U^{v v}\left(r_{i j}\right)$ et vortex-piège $U^{v p}\left(r_{i p}\right)$ où $r_{i p}$ est la distance entre le vortex $i$ et le site de piégeage situé en $\mathbf{r}_{p}$, et $r_{i j}$ est la distance entre les vortex $i$ et $j$ situés à $\mathbf{r}_{i}$ et $\mathbf{r}_{j}$. L'interaction de paire répulsive vortex-vortex est donnée par la fonction de Bessel modifiée $U^{v v}\left(r_{i j}\right)=\alpha_{v} K_{0}\left(r_{i j} / \lambda_{L}\right)$, générant ainsi à l'équilibre un réseau triangulaire de vortex. Le potentiel de piégeage attractif est donné par $U^{v p}\left(r_{i p}\right)=-\alpha_{p} e^{-\left(r_{i p} / R_{p}\right)^{2}}$ où $R_{p}$ est le rayon des pièges. $\alpha_{v}$ et $\alpha_{p}$ sont des paramètres réglables. L'intensité de l'interaction vortex-vortex est fixée en posant $\alpha_{v}=2.8310^{-3} \lambda_{L} \epsilon_{0}$ où $\epsilon_{0}$ est une énergie par unité de longueur. Plusieurs valeurs de l'intensité relative du désordre $\alpha_{p} / \alpha_{v}$ ont été étudiées. Les équations du mouvement sont intégrées par le code classique de dynamique moléculaire LAMMPS [13] avec un algorithme de type Verlet-vitesses. Nous faisons varier la taille du système de $N_{v}=270$ à $N_{v}=12000$ vortex. Diverses formes rectangulaires de la cellule de base de taille $\left(L_{x}, L_{y}\right)$ ont été étudiées, allant de la forme presque carrée $\left(L_{x}, L_{y}\right)=(5,6 \sqrt{3} / 2) n \lambda_{L}$ avec $n=3,8,20$, à des formes très allongées $\left(L_{x}, L_{y}\right)=(400,20 \sqrt{3} / 2) \lambda_{L}$. La géométrie allongée de la cellule de base dans la direction de la force d'entraînement permet l'étude des propriétés critiques au seuil de dépiégeage pour de grands systèmes. Nous avons vérifié qu'une telle anisotropie de la cellule de base ne modifie pas les propriétés critiques et en particulier la détermination de l'exposant critique $\beta$ de la vitesse. Les conditions de bords périodiques sont appliquées dans les deux directions $x$ et $y$. L'interaction vortex-vortex est traitée en utilisant la méthode de la liste de voisins avec un rayon de coupure $r_{c}=6.5 \lambda_{L}$. Le nombre de pièges est tel que $N_{p}=N_{v}$, et leur rayon est $R_{p}=0.22 \lambda_{L}$. Le pas du réseau triangulaire est $a_{0}=\lambda_{L}$. On utilise un système d'unités dans lequel $\lambda_{L}=1$ et $\epsilon_{0}=1$. On fixe $\eta / m=0.1$ où $m$ est la masse du vortex, ce qui permet de retrouver des comportements analogues entre la dynamique du second ordre de Newton et la limite suramortie souvent utilisée pour les vortex supraconducteurs.

\section{DéPiÉgeage Élastique : RÉsultats}

Pour différentes réalisations du désordre, on part du réseau triangulaire de vortex à haute vitesse, avant de décroître lentement la force d'entraînement jusqu'à atteindre la valeur critique du dépiégeage $F_{c}$ en dessous de laquelle les vortex sont tous piégés de manière permanente. Fig. 3 montre l'évolution de la force critique de dépiégeage moyenne mesurée en fonction de l'intensité relative du désordre $\alpha_{p} / \alpha_{v}$ pour $N_{v}=8000$ vortex dans une cellule de base de taille $\left(L_{x}, L_{y}\right)=(400,20 \sqrt{3} / 2) \lambda_{L}$. Comme cela a déjà été montré dans des études de systèmes similaires (voir par exemple Ref. [6]), l'accroissement rapide de la force de dépiégeage indique un changement de régime entre une dynamique élastique dominée par l'élasticité et une dynamique plastique dominée par le désordre.

Nous étudions maintenant le comportement critique à 2D du dépiégeage élastique. Dans la suite du papier, on choisira l'intensité relative du désordre $\alpha_{p} / \alpha_{v} \approx 510^{-3}$ pour étudier la dynamique critique élastique. Fig. 2a montre les trajectoires typiques des vortex au seuil de dépiégeage élastique. Tous les vortex se dépiègent simultanément et avec la même vitesse ce qui implique que chaque vortex garde les mêmes voisins au cours du mouvement. La structure est topologiquement ordonnée et les vortex s'écoulent le long de canaux statiques rugueux et élastiquement couplés. La dynamique correspondante est très saccadée près du seuil et la vitesse du centre de masse des vortex est périodique dans le temps où la période correspond au temps mis par chaque 


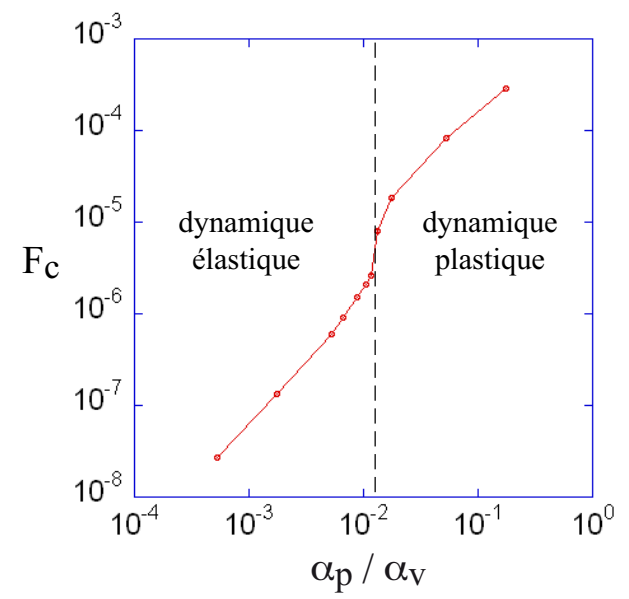

FIgURE 3. Transition entre les régimes dynamiques élastique et plastique mise en évidence par l'évolution de la force critique de dépiégeage moyenne $F_{c}$ en fonction de l'intensité relative du désordre $\alpha_{p} / \alpha_{v}$ pour $N_{v}=8000$ vortex dans une cellule de base de taille $\left(L_{x}, L_{y}\right)=$ $(400,20 \sqrt{3} / 2) \lambda_{L}$.

vortex pour remplacer le vortex qui le précède sur le même canal. Fig. 4 représente la vitesse longitudinale moyenne $v$ du centre de masse des vortex en fonction de la force réduite $f=\left(F-F_{c}\right) / F_{c}$ pour plusieurs tailles de réseau, où $F_{c}$ est la force critique de dépiégeage mesurée pour chaque réalisation du désordre. Trois régions

(a)

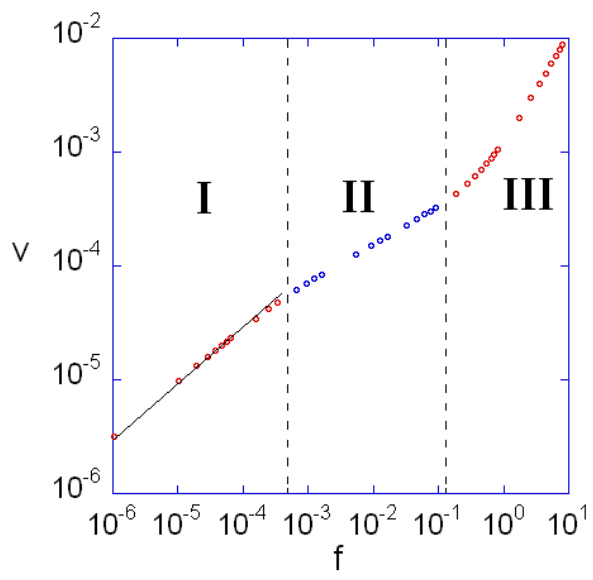

(b)

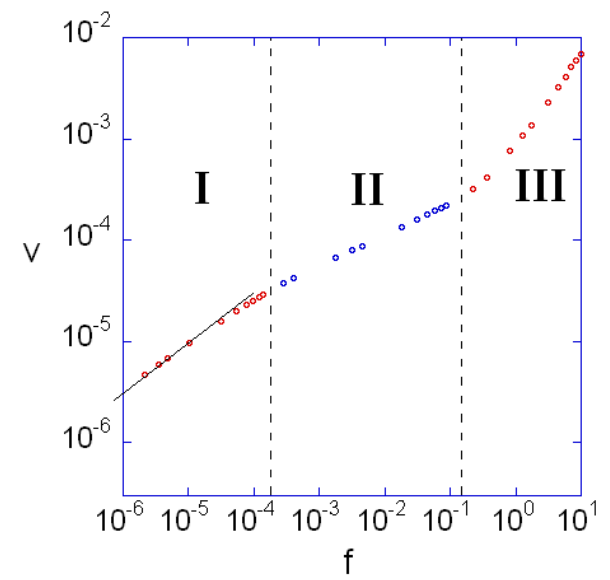

Figure 4. Vitesse $v$ des vortex en fonction de la force réduite $f$ pour différentes tailles de réseau : a) $N_{v}=5000$ vortex dans une cellule de base de taille $\left.\left(L_{x}, L_{y}\right)=(100,50 \sqrt{3} / 2) \lambda_{L} ; \mathrm{b}\right)$ $N_{v}=8000$ vortex dans une cellule de base de taille $\left(L_{x}, L_{y}\right)=(400,20 \sqrt{3} / 2) \lambda_{L}$

apparaissent Fig. 4. La région I est la manifestation des effets de taille finie dans le système simulé dont la signature est le single particle regime [12] où $v \sim f^{1 / 2}$ comme le montrent les lignes de pente $1 / 2$. Dans ce régime, le système se comporte comme une seule particule placée dans un potentiel périodique. Pour $F<F_{c}$ le système possède un point fixe stable (et un instable) correspondant au réseau de vortex piégé. Pour $F=F_{c}$ les deux points fixes entrent en collision lors d'une bifurcation noeud-col et disparaissent pour $F>F_{c}$ pour 
(a)

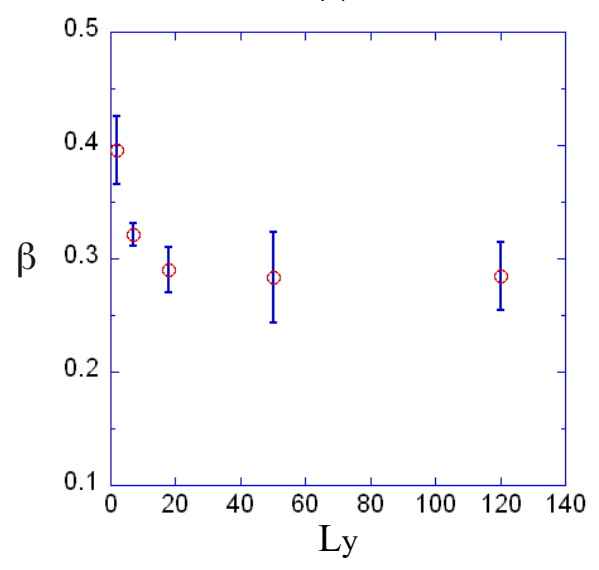

(b)

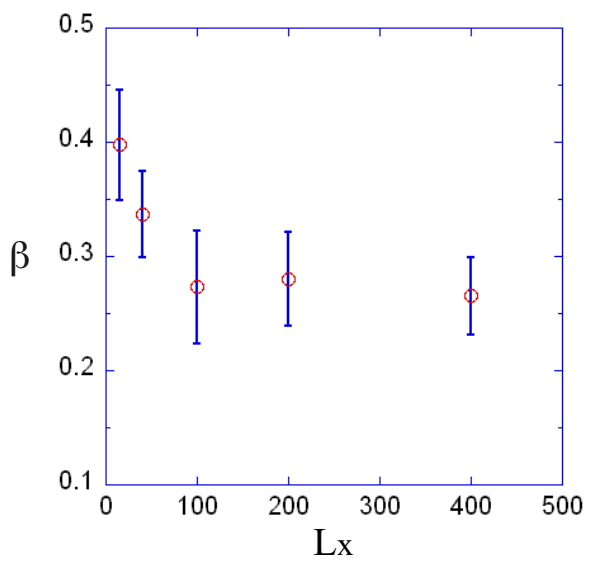

Figure 5. Exposant $\beta$ pour la vitesse : a) en fonction de $L_{y}$ pour $L_{x}=100 \lambda_{L}$ b) en fonction de $L_{x}$ pour diverses tailles transverses de $L_{y}=18 \lambda_{L}$ à $L_{y}=120 \lambda_{L}$. Les barres d'erreurs représentent la déviation standard sur les differentes réalisations du désordre.

donner une dynamique périodique de période $T$ telle que $T \sim f^{-1 / 2}$ lorsque $F \rightarrow F_{c}^{+}$, c'est-à-dire $v \sim f^{1 / 2}$. Dans cette région, un dépiégeage avec hystérésis peut être observé avec différentes valeurs $F_{c}^{c r}$ et $F_{c}^{d e c r}$ du seuil de dépiégeage quand la force croît ou décroît. Néanmoins, la largeur $F_{c}^{c r}-F_{c}^{d e c r}$ de l'hystérésis décroît quand la taille du système simulé augmente, confirmant ainsi que ce phénomène d'hystérésis n'est qu'un effet de taille finie de la simulation numérique et non lié à une transition de phase discontinue (du premier ordre). La région II est celle où une loi puissance $v \sim f^{\beta}$ avec $\beta<1$ est mesurée. Nous identifions donc cette région comme le régime critique de la transition de dépiégeage continue (second ordre). Finalement, dans la région III le système est loin du seuil de dépiégeage et approche asymptotiquement le régime linéaire $v \sim f$ obtenu sans désordre.

Pour renforcer cette image de transition de dépiégeage du second ordre plusieurs tailles du système ont été simulées et pour chaque taille différentes réalisations du désordre sont effectuées à partir desquelles on extrait une valeur moyenne de l'exposant $\beta$ de dépiégeage. Fig. 5a représente l'évolution de $\beta$ en fonction de la taille transverse $L_{y}$ pour une taille longitudinale $L_{x}=100 \lambda_{L}$ fixée. Il apparaît que la valeur de $\beta$ mesurée pour $L_{y} \geq 18 \lambda_{L}$ devient indépendante de la taille transverse $L_{y}$. En particulier, elle ne dépend pas de l'anisotropie de la cellule de base puisque des valeurs identiques sont mesurées pour des cellules de base de forme carrée $\left(L_{x}, L_{y}\right)=(100,120 \sqrt{3} / 2) \lambda_{L}$. Fig. 5 b montre l'évolution de $\beta$ en fonction de la taille longitudinale $L_{x}$ pour diverses tailles transverses $L_{y} \geq 18 \lambda_{L}$. Il apparaît que $\beta$ atteint une valeur constante pour $L_{x} \geq 100 \lambda_{L}$. En prenant la valeur moyenne de $\beta$ sur 68 réalisations du désordre obtenues pour $L_{x} \geq 100 \lambda_{L}$ et $L_{y} \geq 18 \lambda_{L}$, nous obtenons le résultat $\beta=0.27 \pm 0.04$.

Notons que la valeur que nous mesurons est proche de la valeur $\beta \approx 1 / 3$ mesurée pour les interfaces [3] dans un espace de dimension $d=2$. Comparons maintenant nos résultats aux autres systèmes périodiques ayant un champ de déplacement de dimension $N=2$ dans un espace de dimension $d=2$. Par exemple, nos résultats sont proches de la valeur $\beta=0.35$ mesurée récemment dans des systèmes périodiques anisotropes du type stripes [9]. Néanmoins, la variété d'exposants mesurée dans d'autres systèmes périodiques (voir introduction) semble suggérer que les formes précises des interactions particule-particule et particule-piège jouent un rôle important, ce qui impliquerait que de vastes classes d'universalité n'existent pas pour la transition de dépiégeage élastique. Des conclusions similaires émergaient pour la transition de dépiégeage plastique [12]. Finalement, notons que les mesures à température non nulle confirment notre résultat $\beta=0.27 \pm 0.04$. Notons également qu'un travail précédent sur les vortex dans les supraconducteurs en dimension $d=3[14]$ trouvait un exposant 
$\beta=0.65 \pm 0.01$.

\section{Conclusion}

Nous avons présenté des résultats de simulation numérique sur la dynamique de réseaux 2D de vortex supraconducteurs mis en mouvement sur un potentiel désordonné. Un changement de régime entre une dynamique élastique et une dynamique plastique a été mise en évidence, montrant le passage d'un régime dominé par l'élasticité à un régime dominé par le désordre. Dans la phase élastique, nous avons étudié la dynamique au seuil de dépiégeage. Une analogie avec les transitions de phase à l'équilibre du second ordre a été mise en évidence où le paramètre d'ordre est la vitesse et le paramètre de contrôle est la force appliquée. La valeur que nous mesurons pour l'exposant critique $\beta$ associé à la vitesse est $\beta=0.27 \pm 0.04$.

\section{RÉFÉRENCES}

[1] D. S. Fisher, Sliding charge-density waves as a dynamic critical phenomenon, Phys. Rev. B 31 1396 (1985).

[2] S. Bustingorry, A.B. Kolton and T. Giamarchi, Random-manifold to random-periodic depinning of an elastic interface, Phys. Rev. B 82094202 (2010)

A. A. Fedorenko, P. Le Doussal and K. J. Wiese, Universal distribution of threshold forces at the depinning transition, Phys. Rev. E 74041110 (2006).

[3] O. Duemmer and W. Krauth, Critical exponents of the driven elastic string in a disordered medium, Phys. Rev. E 71061601 $(2005)$

U. Nowak and K. D. Usadel, Influence of temperature on the depinning transition of driven interfaces, Europhys. Lett. 44634 (1998)

L. A. N. Amaral, A. L. Barabasi and H. E. Stanley, Universality classes for interface growth with quenched disorder, Phys. Rev. Lett. 7362 (1994).

[4] C. R. Myers and J. P. Sethna, Collective dynamics in a model of sliding charge-density waves. I. Critical behavior, Phys. Rev. B 4711171 (1993)

A. A. Middleton, O. Biham, P. B. Littlewood and P. Sibani, Complete mode locking in models of charge-density waves, Phys. Rev. Lett. 681586 (1992).

[5] G. Piacente and F. M. Peeters, Pinning and depinning of a classic quasi-one-dimensional Wigner crystal in the presence of a constriction, Phys. Rev. B 72205208 (2005).

[6] C. Reichhardt and C. J. Olson, Colloidal dynamics on disordered substrates, Phys. Rev. Lett. 89078301 (2002).

[7] A. Pertsinidis and X. S. Ling, Effects of a random pinning potential on the structure and driven dynamics of two-dimensional colloidal crystals, Bull. Am. Phys. Soc. 46181 (2001).

[8] J. Chen, Y. Cao and Z. Jiao, Dynamics of two-dimensional colloids on a disordered substrate, Phys. Rev. E 69041403 (2004).

[9] C. J. Olson Reichhardt, C. Reichhardt and A. R. Bishop, Anisotropic sliding dynamics, peak effect, and metastability in stripe systems, Phys. Rev. E 83041501 (2011).

[10] E. Olive, J.C. Soret, P. Le Doussal, T. Giamarchi, Numerical simulation evidence of dynamical transverse Meissner effect and moving Bose glass phase, Phys. Rev. Lett. 9137005 (2003).

[11] E. Olive, J.C. Soret, Chaotic dynamics of superconductor vortices in the plastic phase, Phys. Rev. Lett. 96027002 (2006)

E. Olive, J.C. Soret, Chaos and plasticity in superconductor vortices : Low-dimensional dynamics, Phys. Rev. B 77 144514 (2008).

[12] Y. Fily, E. Olive, N. Di Scala and J. C. Soret, Critical behavior of plastic depinning of vortex lattices in two dimensions : Molecular dynamics simulations, Phys. Rev. B 82134519 (2010)

E. Olive, Y. Fily and J. C. Soret, Plastic depinning of superconductor vortices, J. Phys. : Conf. Series 150052201 (2009).

[13] S. Plimpton, Fast parallel algorithms for short-range molecular dynamics, J. Comput. Phys. 1171 (1995).

[14] M. B. Luo and X. Hu, Depinning and creep motion in glass states of flux lines, Phys. Rev. Lett. 98267002 (2007). 